\title{
Some New Results on the Preservation of Stochastic Orders and Aging Classes under Random Minima and Maxima
}

\author{
Ebrahim Salehi* and Ezzatollah Gholami \\ Birjand University of Technology \\ Received: 4/14/2020 Approved: 11/14/2020
}

\begin{abstract}
In this paper, we obtain some results on preservation properties of the moment-generating-function and Laplace transform orders under the taking of random minima and maxima. Also, the reversed preservation of these stochastic orders is studied. In following, we investigate the closure of some new certain aging classes under the random minima and maxima.
\end{abstract}

Keywords. Random minima (maxima); Life distribution class; Series (Parallel) system; Stochastic order; Reliability.

MSC 2010: 60E15, 62N05.

\section{Introduction}

In the recent decades, the properties of maxima and minima of samples, particularly random sized samples is an extensively studied subject. The random sized samples have many real world analogues and applications. For example, if we apply a random censor on life test, the observed data will then be in the form of a random sized sample. There are numerous examples of environmental, agriculture, transportation problems, etc where the maximum and minimum of a sample with random value is considered. Random maxima and minima also have some applications in the study of droughts

${ }^{*}$ Corresponding author 
and floods. Further information regarding these applications can be found in Consul (1984), Shaked and Wong (1997a,b) and Li and Zuo (2004).

An interesting issue of the reliability theory is the preservation of stochastic orders and aging classes under the random maxima and minima. The article of Shaked (1975) on preservation of increasing (decreasing) failure rate; IFR (DFR); life distribution classes under the taking of random maxima and minima is one the earliest works on this subject. The preservation of usual stochastic order, hazard rate order and dispersive order under random maxima and minima are investigated by Shaked and Wong (1997b). Bartoszewicz (2001) extend the results of Shaked and Wong (1997b) for convex transform, star and superadditive orders. He also showed that the increasing (decreasing) failure rate average; IFRA (DFRA); and new better (worse) than used; NBU (NWU); life distribution classes are preserved under the random maxima (random minima), respectively. The preservation of the increasing convex/concave, right spread and total time on test transform orders under the taking random maxima and minima are studied by $\mathrm{Li}$ and Zuo (2004). Furthermore, they investigated the preservation of the increasing failure rate of the second order $(\operatorname{IFR}(2))$ and the new better than used of the second order $(\mathrm{NBU}(2))$ life distribution classes under the random maxima. Li (2004) introduced the $\mathrm{NBU}_{m g f}$ class and showed that this class is closed under increasing star-shaped transformations. Continue Li's research, Ahmad and Kayid (2004) obtained some closure properties and characterizations for $\mathrm{NBU}_{m g f}$ class such as the closure of this class under series systems.

In a study by Ahmad et al. (2005), they discussed the preservation of new better than used in total time on test (NBUT) life distributions class under reliability operations, including random minima. Kayid et al. (2011) studied the closure of the new better (worse) than used in star shaped (NBUS) life distribution class under the formation of a parallel system with a random number of units. The preservation of the new better (worse) than used in convex average; NBUCA (NWUCA); class under reliability operators special random maxima is studied by Ahmad et al. (2006). Other noticeable works on this subject include the article of Hendi et al. (1993), Cai and Wu (1997), Franco et al. (2001, 2003), Nanda et al. (2005), Li and Qiu (2007), Nanda and Shaked (2008) and Miziula (2012). Later studies such as the one published by Hazra et al. (2014) corrected some wrong conclusions of previous works and provided some new results regarding preservation of life distribution classes under series and parallel systems and even $k$-out-of- $n$ 
systems with random number of components.

In addition to these studies, some researchers examined the reversed preservation of life distribution classes for random maxima and minima. Li and Yam (2005) studied the reversed preservation of negative aging classes and showed that when the lifetime of a parallel system belong to new worse than used in second order; $\mathrm{NWU}(2)$; class, the lifetime of its components are also NWU(2). They also proved that when the lifetime of series system is new worse than used in convex ordering; NWUC; and increasing (decreasing) mean residual lifetime; IMRL (DMRL); the lifetime of its components are also of the same class (NWUC or IMRL). Ahmad et al. (2006) showed that when the lifetime of a series system belong to NWUCA class, the lifetime of its components are also NWUCA. Belzunce et al. (2007) also studied the reversed preservation of IMRL, new better than used in expectation; NWUE; and DFR under the parallel system, and proved when the lifetime of a parallel system is of one of those three classes, the lifetime of its components are also of the same class; they also presented some results regarding the reversed preservation of IFRA (DFRA) and NBUC under the series system. Other attempts on this topic can be found in Ahmad and Kayid (2007).

The study of order statistic has attracted a great deal of attention from both theoretical and practical perspectives. Let $X_{1}, X_{2}, \ldots, X_{n}$ be $n$ random variables. If the $X$ 's are arranged in ascending order of magnitude then we have $X_{1: n} \leq X_{2: n} \leq \ldots \leq X_{n: n} . \quad X_{i: n}$ is called the $i$ th order statistic of $X_{1}, X_{2}, \ldots, X_{n}, i=1,2, \ldots, n$. For a comprehensive reference on order statistics we refer the reader to David and Nagaraja (2003). One of the topics that has many applications in the theory of reliability, survival analysis, economics, etc. is stochastic comparisons between probability distributions. Many researchers have investigated stochastic comparisons of order statistics restricted to cases whereby the random variables are independent. Recently, the attention of researchers has focused to study this topic in the dependence case. Among others, we imply to Boland et al. (1994), Hu and He (2000), Belzunce et al. (2001), Nanda et al. (2005), Kochar (2012) and Salehi and Tavangar (2019) and references within it.

Applications and properties of the moment generating function order and Laplace transform order can be found in Alzaid et al. (1991), Denuit, M. (2001), Klar and Muller (2003), Li (2004), Ahmad and Kayid (2004), Shaked and Shanthikumar (2007), Jarrahiferiz et al. (2016) and Zamani et al. (2017). A noticeable research of application of the moment generating function order is made by Kaas and Hesselager (1995). They used the mo- 
ment generating function order to obtain some significant results that might be used to model claim sizes. The aim of the present paper is to show that the moment-generating-function and Laplace transform orders are preserved (reversed preserved) under random maxima (random minima). The other aim of the paper is to extend the results of Hazra et al. (2014) to new aging classes.

The paper is organized as follows. In Section 2, we provide some concepts and necessary preliminaries. Beside, we present some definitions of life distribution classes. The preservation and reversed preservation of the moment generating function order and Laplace transform order under random minima and maxima are studied in Section 3. Finally, in Section 4, we discuss the closure of some aging properties under random maxima and minima with illustrative counter-examples.

\section{Preliminaries}

This section introduces a number of definitions and concepts that are important for presenting the conclusions in the following sections. First, we introduce two important criteria that play a key role in reliability theory and survival analysis.

Let $X$ be a non-negative random variable with distribution function $F$, density function $f$ and reliability function $\bar{F}=1-F$. The hazard function of $X$, denoted by $h$, is defined as

$$
h(t)=\frac{f(t)}{\bar{F}(t)} .
$$

In fact, the hazard function represents the failure rate of a unit or the effect of age and passage of time on the survival of a living unit. Therefore, this function is a good criterion for measuring aging. The integral of the hazard rate function over the interval $(0, t]$ is called the cumulative hazard function, which is denoted by $H$, i.e

$$
H(t)=\int_{0}^{t} h(u) d u=-\log \bar{F}(t) .
$$

The residual lifetime of $X$ at age $t \geq 0$, denoted by $X_{t}$, is the remaining 
life of $X$ given survival at age $t$, that is,

$$
X_{t}=\{X-t \mid X>t\}
$$

The reliability function of $X_{t}$, denoted by $\bar{F}_{t}$, is

$$
\bar{F}_{t}(x)=\frac{\bar{F}(x+t)}{\bar{F}(t)}, \quad x \geq 0 .
$$

The second concept is the mean residual life (MRL) function, denoted by $m(t)$, which is one of the most important and commonly used criteria in the reliability theory. This function is defined as:

$$
m(t)=E(X-t \mid X>t)=\frac{1}{\bar{F}(t)} \int_{t}^{\infty} \bar{F}(x) d x .
$$

In the following, we present the definition of some concepts of stochastic orders which will be used throughout the paper. For more details on stochastic orders see Shaked and Shanthikumar (2007).

Definition 1. Let $X$ and $Y$ two non-negative random variables with continuous distribution functions $F(x)$ and $G(x)$, and survival functions $\bar{F}(x)$ and $\bar{G}(x)$, respectively. $X$ is said to be smaller than $Y$ in

i) the usual stochastic order, denoted by $X \leq_{s t} Y$, if for all $x, \bar{F}(x) \leq$ $\bar{G}(x)$;

ii) the increasing concave order, denoted by $X \leq_{i c v} Y$, if

$$
\int_{0}^{t} \bar{F}(x) d x \leq \int_{0}^{t} \bar{G}(x) d x
$$

iii) the moment-generating-function order, denoted by $X \leq_{m g f} Y$, if for all $s \geq 0$,

$$
\int_{0}^{\infty} e^{s x} \bar{F}(x) d x \leq \int_{0}^{\infty} e^{s x} \bar{G}(x) d x .
$$

iv) the Laplace transform order, denoted by $X \leq_{l t} Y$, if for all $s \geq 0$,

$$
\int_{0}^{\infty} e^{-s x} \bar{F}(x) d x \leq \int_{0}^{\infty} e^{-s x} \bar{G}(x) d x .
$$


The life distribution classes, each of which representing a distinctive property of life distributions, play a significant role in reliability studies. Most life distribution classes are defined and classified based on the concept of hazard rate and stochastic orders. Barlow and Proschan (1975) and Lai and Xie (2006) have provided full and comprehensive literature reviews on the subject of life distribution classes. Some of the most important life distribution classes are described in the following.

Definition 2. Let $X$ be a non-negative random variable with distribution function $F . X$ (or $F$ ) is said to be

i) IFR (DFR), if and only if for all $x \geq 0, \bar{F}_{t}(x)$ is decreasing (increasing) function in $t$.

ii) $\operatorname{IFR}(2)(\operatorname{DFR}(2))$, if and only if for all $x \geq 0$,

$$
\int_{0}^{x} \frac{\bar{F}\left(u+t_{2}\right)}{\bar{F}\left(t_{2}\right)} d u \leq(\geq) \int_{0}^{x} \frac{\bar{F}\left(u+t_{1}\right)}{\bar{F}\left(t_{1}\right)} d u, \quad 0 \leq t_{1}<t_{2} .
$$

iii) NBUT (NWUT), if and only if for all $p \in(0,1)$,

$$
\int_{0}^{F_{t}^{-1}(p)} \bar{F}(u+t) d u \leq(\geq) \bar{F}(t) \int_{0}^{F_{t}^{-1}(p)} \bar{F}(u) d u d x .
$$

iv) $\mathrm{NBU}(2)(\mathrm{NWU}(2))$, if and only if for all $x, t \geq 0$,

$$
\int_{0}^{x} \bar{F}(u+t) d u \leq(\geq) \bar{F}(t) \int_{0}^{x} \bar{F}(u) d u d x .
$$

v) $\mathrm{NBU}(3)(\mathrm{NWU}(3))$ if and only if for all $t \geq 0$,

$$
\int_{0}^{\infty} \int_{0}^{x} \bar{F}(u+t) d u d x \leq(\geq) \bar{F}(t) \int_{0}^{\infty} \int_{0}^{x} \bar{F}(u) d u d x
$$

vi) NBUS (NWUS), if and only if for all $t, s \geq 0$,

$$
\int_{s}^{\infty} x f(t+x) d x \leq(\geq) f(t) \int_{s}^{\infty} f(x) d x
$$


vii) $\mathrm{NBU}_{m g f}\left(\mathrm{NWU}_{m g f}\right)$, if and only if for all $t, s \geq 0$,

$$
\int_{0}^{\infty} e^{s x} \bar{F}(x+t) d x \leq(\geq) \bar{F}(t) \int_{0}^{\infty} e^{s x} \bar{F}(x) d x
$$

viii) new better (worse) than used in Laplace transform, denoted by NBUL (NWUL), if and only if for all $t, s \geq 0$,

$$
\int_{0}^{\infty} e^{-s x} \bar{F}(x+t) d x \leq(\geq) \bar{F}(t) \int_{0}^{\infty} e^{-s x} \bar{F}(x) d x
$$

ix) NBUCA (NWUCA) if and only if for all $t \geq 0$,

$$
\int_{0}^{\infty} \int_{x}^{\infty} \bar{F}(u+t) d u d x \leq(\geq) \bar{F}(t) \int_{0}^{\infty} \int_{x}^{\infty} \bar{F}(u) d u d x .
$$

$\mathrm{x})$ new better than used failure rate, denoted by NBUFR (NWUFR), if and only if for all $t \geq 0$,

$$
\lim _{x \rightarrow 0^{+}} \frac{1}{x} \int_{0}^{x} h(u) d u \leq(\geq) h(t) .
$$

xi) new better than average failure rate, denoted by NBAFR (NWAFR), if and only if for all $t \geq 0$,

$$
h(0) \leq(\geq)-\frac{1}{t} \log \bar{F}(t) .
$$

Here, we present the relationships among some positive aging classes in Fig. 1. The relations among negative aging classes is similar.

Before stating our main conclusions, we also recall the following required lemmas which will be frequently used in sequel.

Lemma 1. Given a non-negative integer $n$, than

i) (Li and Lu (2003)):

$$
\left(\max \left\{X_{1}, X_{2}, \ldots, X_{n}\right\}\right)_{t} \leq_{s t} \max \left\{\left(X_{1}\right)_{t},\left(X_{2}\right)_{t}, \ldots,\left(X_{n}\right)_{t}\right\} ;
$$

ii) (Pellerey and Petakos (2002)):

$$
\min \left\{\left(X_{1}\right)_{t},\left(X_{2}\right)_{t}, \ldots,\left(X_{n}\right)_{t}\right\}={ }_{s t}\left(\min \left\{X_{1}, X_{2}, \ldots, X_{n}\right\}\right)_{t} .
$$




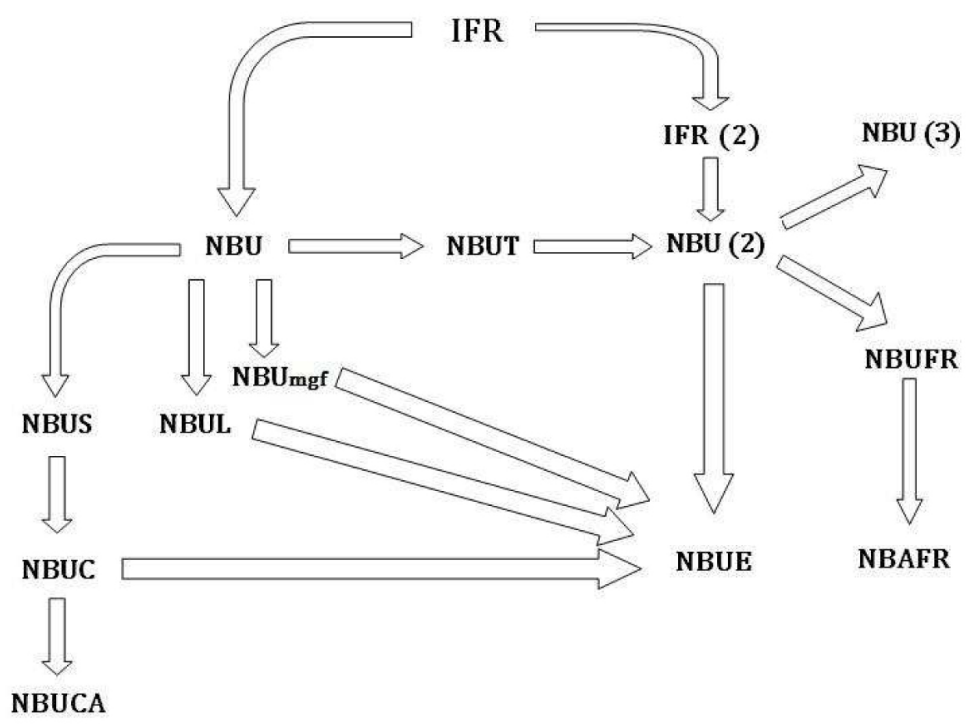

Figure 1. The relationships among positive aging classes.

Lemma 2. (Barlow and Proschan (1975)) Let $W(x)$ be a Lebesgue-Stieltjes measure, not necessarily positive, for which $\int_{t}^{\infty} d W(x) \geq 0$ for all $t$, and let $h(x) \geq 0$ be increasing. Then

$$
\int_{-\infty}^{\infty} h(x) d W(x) \geq 0
$$

\section{Preservation of Stochastic Orders}

Let $\left\{X_{n}\right\}_{n=1}^{\infty}$ be a sequence of independent and identical random variables with absolutely continuous distribution $F$ and $N$ be a positive integervalued random variable which is independent of the $X_{i}$ 's. The lifetimes of a parallel system with the random number $N$ of components, i.e. $X_{N: N}=$ $\max \left\{X_{1}, X_{2}, \ldots, X_{N}\right\}$, has the reliability function as follow:

$$
\bar{F}_{N: N}(t)=\sum_{n=1}^{\infty}\left[1-F^{n}(t)\right] p_{N}(n),
$$


where $p_{N}(n)$ is the probability mass function of the random variable $N$. Also, the reliability function of $X_{1: N}=\min \left\{X_{1}, X_{2}, \ldots, X_{N}\right\}$, that is the lifetimes of a series system with the random number $N$ of components, is equal to

$$
\bar{F}_{1: N}(t)=P\left(X_{1: N}>t\right)=\sum_{n=1}^{\infty} \bar{F}^{n}(t) p_{N}(n) .
$$

First, the following theorem gives the preservation of the moment-generatingfunction and Laplace transform orders under random maxima.

Theorem 1. Let $\left\{X_{n}\right\}_{n=1}^{\infty}$, be a sequence of i.i.d random variables with distribution function $F$, and $\left\{Y_{n}\right\}_{n=1}^{\infty}$, be another sequence of i.i.d random variables with distribution function $G$. Also, assume that $N$ is independent of $X_{i}$ 's and $Y_{i}$ 's.

i) If $X_{1} \leq_{m g f} Y_{1}$, then $X_{N: N} \leq_{m g f} Y_{N: N}$;

ii) if $X_{1} \leq_{l t} Y_{1}$, then $X_{N: N} \leq_{l t} Y_{N: N}$.

Proof. Let us assume that $X_{1} \leq_{m g f} Y_{1}$. It implies that for all $s \geq 0$,

$$
\int_{0}^{\infty} e^{s x}[\bar{G}(x)-\bar{F}(x)] d x=\int_{0}^{\infty} e^{s x}[F(x)-G(x)] d x \geq 0 .
$$

On the other hand, we have for all $s \geq 0$,

$$
\begin{aligned}
\int_{0}^{\infty} e^{s x}\left[F_{N: N}(x)-G_{N: N}(x)\right] d x & =\int_{0}^{\infty} e^{s x} \sum_{n=1}^{\infty} p_{N}(n)\left[F^{n}(x)-G^{n}(x)\right] d x \\
& =\int_{0}^{\infty} \Delta(x) e^{s x}[F(x)-G(x)] d x \\
& \geq 0
\end{aligned}
$$

where

$$
\Delta(x)=\sum_{n=1}^{\infty} p_{N}(n) \sum_{j=0}^{n-1}[F(x)]^{n-j-1} G^{j}(x),
$$

is non-negative and increasing function. Note that the last inequality is obtained from (3) and Lemma 2. Therefore, $X_{N: N} \leq_{m g f} Y_{N: N}$ and case i) is proved. The proof of case ii) is similar to the proof case $i$ ), and details are omitted. 
Theorem 2. Let $\left\{X_{n}\right\}_{n=1}^{\infty}$ and $\left\{Y_{n}\right\}_{n=1}^{\infty}$ be two sequences of i.i.d random variables with distribution function $F$ and $G$, respectively. Assume that $N$ is independent of $X_{i} s$ and $Y_{i}$ s.

i) If $X_{1: N} \leq_{m g f} Y_{1: N}$, then $X_{1} \leq_{m g f} Y_{1}$;

ii) if $X_{1: N} \leq_{l t} Y_{1: N}$, then $X_{1} \leq_{l t} Y_{1}$.

Proof. Let us consider only the case i), because the other case is analogous. $X_{1: N} \leq_{m g f} Y_{1: N}$ means that for all $s \geq 0$,

$$
\int_{0}^{\infty} e^{s x}\left[\bar{G}_{1: N}(x)-\bar{F}_{1: N}(x)\right] d x \geq 0 .
$$

However, for all $s \geq 0$,

$$
\begin{aligned}
\int_{0}^{\infty} e^{s x}\left[\bar{G}_{1: N}(x)-\bar{F}_{1: N}(x)\right] d x & =\int_{0}^{\infty} e^{s x} \sum_{n=1}^{\infty} p_{N}(n)\left[\bar{G}^{n}(x)-\bar{F}^{n}(x)\right] d x \\
& =\int_{0}^{\infty} \Delta^{*}(x) e^{s x}[\bar{G}(x)-\bar{F}(x)] d x
\end{aligned}
$$

where

$$
\Delta^{*}(x)=\sum_{n=0}^{\infty} p_{N}(n) \sum_{j=0}^{n-1}[\bar{F}(x)]^{n-j-1} \bar{G}^{j}(x) .
$$

Note that $\left[\Delta^{*}(x)\right]^{-1}$ is non-negative and increasing function. Hence, using Lemma 2 we conclude that

$$
\int_{0}^{\infty} e^{s x}[\bar{G}(x)-\bar{F}(x)] d x \geq 0
$$

that is to say $X_{1} \leq_{m g f} Y_{1}$. Therefore, the proof is complete.

Corollary 1. Let $X_{1}, X_{2}, \ldots, X_{n}\left(Y_{1}, Y_{2}, \ldots, Y_{n}\right)$ be a set of $n$ i.i.d random variable with distribution function $F(G)$. From Theorems 1 and 2 we have

i) If $X_{1} \leq_{m g f}\left(\leq_{l t}\right) Y_{1}$, then $X_{n: n} \leq_{m g f}\left(\leq_{l t}\right) Y_{n: n}$;

ii) if $X_{1: n} \leq_{m g f}\left(\leq_{l t}\right) Y_{1: n}$, then $X_{1} \leq_{m g f}\left(\leq_{l t}\right) Y_{1}$.

Remark 1. Shaked and Shanthikumar (2007) showed that if $X_{i} \leq_{l t} Y_{i}$, and $\bar{F}_{i}$ and $\bar{G}_{i}, i=1,2, \ldots, n$, are completely monotone, then $X_{1: n} \leq_{l t} Y_{1: n}$. 


\section{Preservation of Aging Classes}

In this section we study the preservation of some certain aging classes under the random minima and maxima. Suppose $X_{1}, X_{2}, \ldots, X_{n}$ are random variables and $X_{1: n}, X_{2: n}, \ldots, X_{n: n}$ are their corresponding order statistics. Sometimes the number of variables is itself a random variable. In that case, the corresponding order statistics are represented by $X_{1: N} \leq X_{2: N} \leq \ldots \leq$ $X_{N: N}$, where $N$ is a positive integer denoting the number of random variables. In the following, we first examine the preservation of some aging classes under random maxima. The preservation of positive aging classes under random minima is discussed in Subsection 4.2.

\subsection{Random Maxima}

$\mathrm{Li}$ and $\mathrm{Lu}$ (2003) showed that the following stochastic inequality is true for $t>0$ :

$$
\left(\max \left\{X_{1}, X_{2}, \ldots, X_{n}\right\}\right)_{t} \leq_{s t} \max \left\{\left(X_{1}\right)_{t},\left(X_{2}\right)_{t}, \ldots,\left(X_{n}\right)_{t}\right\} .
$$

Using Equation (4), they proved that IFR and DFR life distribution classes are preserved under the formation of series and parallel systems with $n$ independent and identically distributed components. In a study by Li and Zuo (2004), Equation (4) was generalized for when $n$ is replaced with the random positive integer $N$, and it was concluded that the life distribution classes that are closed under minima and maxima are also closed under random minima and maxima, that is, series and parallel systems with a random number $N$ components. But as Hazra et al. (2014) have shown, this conclusion is incorrect, meaning that

$$
\left(\max \left\{X_{1}, X_{2}, \ldots, X_{N}\right\}\right)_{t} \underline{\perp}_{s t} \max \left\{\left(X_{1}\right)_{t},\left(X_{2}\right)_{t}, \ldots,\left(X_{N}\right)_{t}\right\} .
$$

Hazra et al. (2014) provided a counterexample demonstrating that some life distribution classes are not closed under random maxima. Here, we show that the life distribution classes IFR(2), NBUS, NBUT, NBU(2), NBUFR, NBAFR, and NBUCA are also not preserved under random maxima.

Example 4. Let $X_{1}, \ldots, X_{N}$ be independent standard exponential random variables and let $N$ be an integer-valued random variable with probability 
mass function

$$
P(N=1)=0.99, \quad P(N=7)=0.01 .
$$

Also, assume that $N$ is independent of $X_{i}$ 's. It is clear that $X_{i}$ 's are IFR. Hence, from Fig. $1, X_{i}$ 's are NBUS and NBUCA. Using (1), we have

$$
\bar{F}_{N: N}(t)=0.99 \exp \{-t\}+0.01\left(1-(1-\exp \{-t\})^{7}\right) .
$$

Now, we show that $\bar{F}_{N: N}$ isn't NBUCA. For this purpose first we compute the below expressions for $t \geq 0$. Therefore,

$$
\begin{aligned}
& A(t)=\frac{1}{\bar{F}_{N: N}(t)} \int_{0}^{\infty} \int_{x}^{\infty} \bar{F}_{N: N}(u+t) d u d x \\
& =\frac{1}{\bar{F}_{N: N}(t)} \int_{0}^{\infty}\left(0.99 e^{-(x+t)}+0.01\left[7 e^{-(x+t)}-\frac{21}{2} e^{-2(x+t)}+\frac{35}{3} e^{-3(x+t)}\right.\right. \\
& \left.\left.-\frac{35}{4} e^{-4(x+t)}+\frac{21}{5} e^{-5(x+t)}-\frac{7}{6} e^{-6(x+t)}+\frac{1}{7} e^{-7(x+t)}\right]\right) d x \\
& =\frac{1}{\bar{F}_{N: N}(t)}\left(0.99 e^{-t}+0.01\left[7 e^{-t}-\frac{21}{4} e^{-2 t}\right.\right. \\
& \left.\left.+\frac{35}{9} e^{-3 t}-\frac{35}{16} e^{-4 t}+\frac{21}{25} e^{-5 t}-\frac{7}{36} e^{-6 t}+\frac{1}{49} e^{-7 t}\right]\right),
\end{aligned}
$$

and also,

$$
\begin{aligned}
B & =\int_{0}^{\infty} \int_{x}^{\infty} \bar{F}_{N: N}(u) d u d x \\
& =\int_{0}^{\infty}\left(0.99 e^{-x}+0.01\left[7 e^{-x}-\frac{21}{2} e^{-2 x}+\frac{35}{3} e^{-3 x}\right.\right. \\
& \left.\left.\quad-\frac{35}{4} e^{-4 x}+\frac{21}{5} e^{-5 x}-\frac{7}{6} e^{-6 x}+\frac{1}{7} e^{-7 x}\right]\right) d x \\
& =1.0312 .
\end{aligned}
$$

In Fig. 2, the function $\mathrm{A}(\mathrm{t})$ is plotted against the constant $\mathrm{B}$. As this figure shows, for $t \in(0,0.6735)$, we have $A(t)>B$. Therefore, according to Definition 2 (ix), $F_{N: N}$ does not belong to the NBUCA class. According to Figure $1, F_{N: N}$ does not belong to the NBUS class either, which means that these classes are not closed under random maxima. 


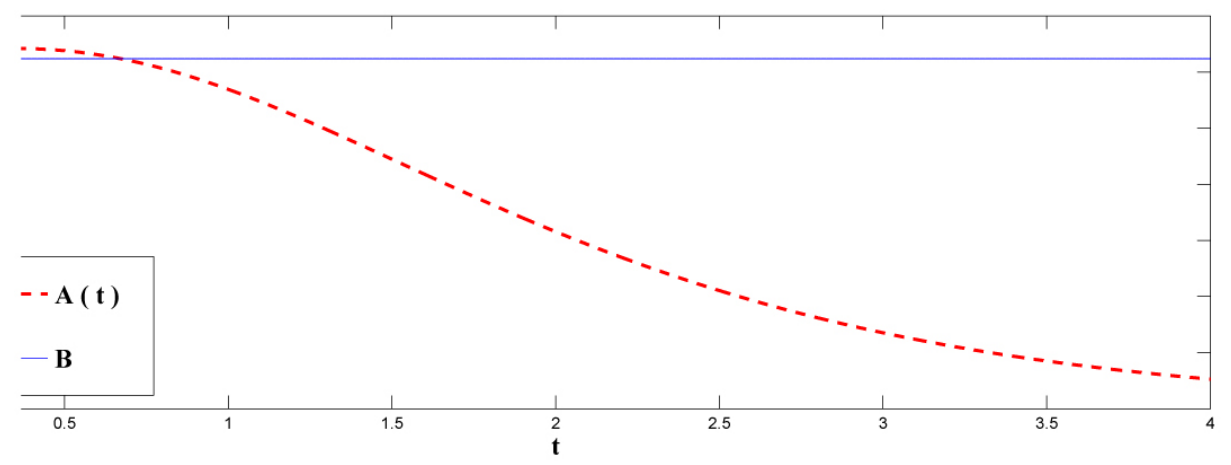

Figure 2. The graphs of function $\mathrm{A}(\mathrm{t})$ and $\mathrm{B}$ in Example 4.

Remark 2. Ahmad et al. (2006) and Kayid et al. (2011) used the below stochastic order

$$
\left(\max \left\{X_{1}, X_{2}, \ldots, X_{N}\right\}\right)_{t} \leq_{s t} \max \left\{\left(X_{1}\right)_{t},\left(X_{2}\right)_{t}, \ldots,\left(X_{N}\right)_{t}\right\},
$$

which is generally incorrect, to argue that the NBUCA and NBUS life distribution classes are preserved under random maxima, respectively. But as Example 4 demonstrates, this conclusion is incorrect. In other words, NBUCA and NBUS are not preserved under random maxima.

Example 5. Let $X_{1}, \ldots, X_{N}$ be independent and identical with exponential random variables and let $N$ be an integer-valued random variable with probability mass function

$$
P(N=1)=0.85, \quad P(N=5)=0.15 .
$$

Furthermore, assume that $N$ is independent of $X_{i}$ 's. It is clear that $X_{i}$ 's are IFR and hence they are also IFR(2), NBUT, NBU(2), NBUFR, and NBAFR. From equation (1),

$$
\bar{F}_{N: N}(t)=0.85 \exp \{-2 t\}+0.15\left(1-(1-\exp \{-2 t\})^{5}\right) .
$$

In the following, we show that $F_{N: N}$ does not belong to the NBAFR class. To do so, we first compute the hazard rate function and the cumulative hazard function of a parallel system with a random number of units. Thus, 


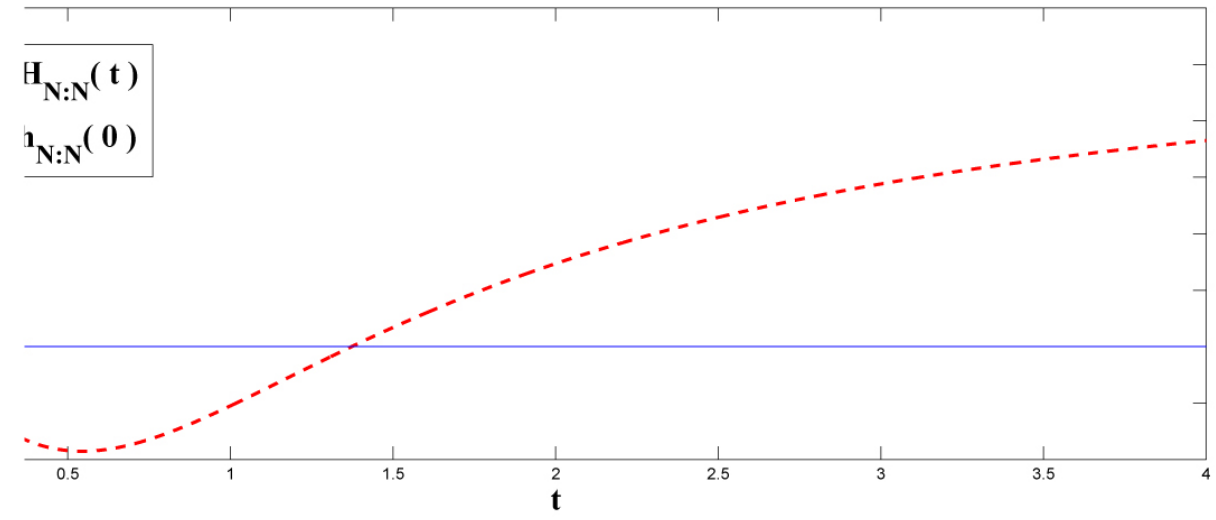

Figure 3. The graphs of $h_{N: N}(0)$ and $H_{N: N}(t)$ for random maxima in Example 5 .

we have

$$
\begin{aligned}
h_{N: N}(t) & =\frac{f_{N: N}(t)}{\bar{F}_{N: N}(t)} \\
& =\frac{1.7 \exp \{-2 t\}+1.5 \exp \{-2 t\}(1-\exp \{-2 t\})^{4}}{0.85 \exp \{-2 t\}+0.15\left[1-(1-\exp \{-2 t\})^{5}\right]},
\end{aligned}
$$

and

$$
\begin{aligned}
H_{N: N}(t) & =-\frac{1}{t} \log \bar{F}_{N: N}(t) \\
& =-\frac{1}{t} \log \left(0.85 \exp \{-2 t\}+0.15\left[1-(1-\exp \{-2 t\})^{5}\right]\right) .
\end{aligned}
$$

The curves of the functions $h_{N: N}(0)=1.7$ and $H_{N: N}(t)$ are plotted in Fig. 3. From this figure and Definition 2, (xi), it can be concluded that $F_{N: N}$ does not belong to the NBAFR class, which means that the NBAFR class is not preserved under random maxima. Therefore, considering the relationship between the life distribution classes in Fig. 1, the IFR(2), NBUT, NBU(2) and NBUFR classes are also not closed under random maxima.

In the following, we show that the $\mathrm{NBU}_{m g f}$ and NBUL classes are preserved under the parallel system consisting of independent and identical components. 
Theorem 3. Suppose that $X_{1}, X_{2}, \ldots, X_{n}$ are i.i.d non-negative random variables with distribution function $F$. If $F$ is $N B U_{m g f}(N B U L)$, then $F_{n: n}$ is also $N B U_{m g f}(N B U L)$.

Proof. $X$ is $\mathrm{NBU}_{m g f}$ if and only if $X_{t} \leq_{m g f} X$ for all $t \geq 0$. Using Corollary 1 case i), we deduce

$$
\max \left\{\left(X_{1}\right)_{t},\left(X_{2}\right)_{t}, \ldots,\left(X_{n}\right)_{t}\right\} \leq_{m g f} X_{n: n} .
$$

Since, $\leq_{s t}$ implies that $\leq_{m g f}$ and using Lemma 1 case i), we have for all $t \geq 0,\left(X_{n: n}\right)_{t} \leq_{m g f} X_{n: n}$, which gives the required result. The proof for the NBUL class is similar, and the details are omitted.

Remark 3. Using the Counter-Example 4.1 in Hazra et al. (2014), one can deduce that the $\mathrm{NBU}_{m g f}$ and NBUL classes are not closed under the formation of random maxima.

In below, with a counter-example we show that negative aging (life distribution) classes $\operatorname{DFR}(2), \mathrm{NWU}(2), \mathrm{NWUS}, \mathrm{NWUT}, \mathrm{NWU}_{m g f}$, NWUL, NWUFR, NWAFR and NWUCA are not preserved under random maxima. For this purpose, in the following example we assume that the random variable $N$ is degenerated in value 2 .

Example 6. Suppose $X_{1}$ and $X_{2}$ are independent and identically distributed random variables representing the lifetime of two components, which have an exponential distribution with $\lambda=1$. Since exponential distribution belongs to the DFR class (for more details, see Barlow and Proschan (1975)), given the relationship between the negative aging classes (as in Fig. 1), this distribution also belongs to the $\operatorname{DFR}(2)$, NWU(2), NWUS, NWUT, NWUFR, NWAFR and NWUCA classes. Now, consider a parallel system composed of these two components. The distribution function and the hazard rate function of the system lifetime will be:

$$
F_{2: 2}(t)=(1-\exp \{-t\})^{2},
$$

and then

$$
h_{2: 2}(t)=1-\frac{1}{2 \exp \{t\}-1}
$$


According to Definition 2 (ix), for all $t \geq 0$, we have

$$
\begin{aligned}
& \int_{0}^{\infty} \int_{x}^{\infty} \bar{F}_{2: 2}(u+t) d u d x-\bar{F}_{2: 2}(t) \int_{0}^{\infty} \int_{x}^{\infty} \bar{F}_{2: 2}(u) d u d x \\
= & \int_{0}^{\infty}\left(2 \exp \{-(x+t)\}-\frac{1}{2} \exp \{-2(x+t)\}\right) d x \\
& -(2 \exp \{-t\}-\exp \{-2 t\}) \int_{0}^{\infty}\left(2 \exp \{-x\}-\frac{1}{2} \exp \{-2 x\}\right) d x \\
= & \frac{3}{2}(\exp \{-2 t\}-\exp \{-t\}) \\
\leq & 0,
\end{aligned}
$$

This indicates that $F_{2: 2}$ does not belong to the NWUCA class, and given the relationship between NWUS and NWUCA (as in Fig. 1), it also does not belong to NWUS. Therefore, this class is not preserved under maxima. From this, it can be concluded that the NWUS and NWUCA classes will not be preserved under random maxima, either.

It is easy to show that $h_{2: 2}(0)=0$. For all $t \geq 0$, we have

$$
H_{2: 2}(t)=-\frac{1}{t} \log \bar{F}_{2: 2}(t) \geq h_{2: 2}(t)=0 .
$$

Thus, according to the Definition 2 , the above inequality implies that $F_{2: 2}$ does not belong to the NWAFR class and then $\operatorname{DFR}(2)$, NWU(2), NWUT and NWUFR, too. Therefore, above listed classes are not preserved under random maxima.

Remark 4. It is known that the maximum of two independent standard exponential random variables is not NWUE (Barlow and Proschan (1975), p. 183). Since the NWU $m g f$ and NWUL are subclasses of NWUE, then $\mathrm{NWU}_{m g f}$ and NWUL classes are not closed under the formation of maxima. Therefore, these classes are not preserved under the random maxima.

\subsection{Random Minima}

In this section we focus on investigating the preservation of life distribution classes under random minima. Let $\left\{X_{1}, X_{2}, \ldots\right\}$ be a sequence of independent and identical random variables with absolutely continuous distribution $F$ and $N$ be a positive integer-valued random variable which is independent of the $X_{i}$ s. 
According to Remark 4.4 in Hazra et al. (2014), we can be shown that life distribution classes $\operatorname{IFR}(2), \mathrm{NBU}(2), \mathrm{NBU}(3), \mathrm{NBU}_{m g f}$, NBUL, NBUS, NBUT, NBUFR, NBAFR and NBUCA are not preserved under random minima.

Example 7. Consider independent and identical random variables $X_{1}, \ldots, X_{N}$ that have standard exponential distribution. Let $N$ be an integer-valued random variable having support in $\{1,2\}$, and independent of $X_{1}$ and $X_{2}$, such that

$$
P(N=1)=1-P(N=2)>0 .
$$

As mentioned by Hazra et al. (2014), $F_{1: N}$ is a mixture of the distribution of $X_{1: 1}={ }_{s t} X_{1}$ and the distribution of $X_{1: 2}$. It is known that the distribution of $X_{1: 2}$ is exponential with parameter 2. On the other hands, Barlow and Proschan (1975) (in page 103 and also, page 186) implied that the mixture of two non-identical exponential distributions has a strictly decreasing hazard rate function. Hence, $F_{1: N}$ cannot be $\operatorname{IFR}(2), \mathrm{NBU}(2), \mathrm{NBU}(3), \mathrm{NBU}_{m g f}$, NBUL, NBUS, NBUT, NBUFR, NBAFR or NBUCA. It means that these classes are not closed under the formation of random minima.

Remark 5. Li and Zuo (2004) and Ahmad et al. (2005) used the relationship

$$
\min \left\{\left(X_{1}\right)_{t},\left(X_{2}\right)_{t}, \ldots,\left(X_{N}\right)_{t}\right\}={ }_{s t}\left(\min \left\{X_{1}, X_{2}, \ldots, X_{N}\right\}\right)_{t}
$$

which is generally incorrect, to argue that the $\operatorname{IFR}(2)$ and NBUT classes are preserved under random minima, respectively. As shown in Example 7, this conclusion is incorrect, meaning that the $\operatorname{IFR}(2)$ and NBUT classes are not preserved under random minima.

However, in the next result we show that when the lifetime of series system consisting of $n$ i.i.d components is $\mathrm{NBU}_{m g f}$ (NBUL), the lifetime of its components is also $\mathrm{NBU}_{m g f}$ (NBUL).

Proposition 1. Suppose that $X_{1}, X_{2}, \ldots, X_{n}$ are i.i.d non-negative random variables with distribution function $F$. If $F_{1: n}$ is $\mathrm{NBU}_{m g f}$ (NBUL), then $F$ is also $\mathrm{NBU}_{m g f}(\mathrm{NBUL})$.

Proof. Using Lemma 1 (ii) and Corollary 1 (ii), the proof is complete. 


\section{Discussions \& Conclusions}

Many researchers have shown interest in the properties of life distribution classes under random maxima and minima. In this paper, we first showed that the moment-generating-function and Laplace transform orders are preserved under random maxima and then studied the preservation of these stochastic orders under random minima. Afterward, we investigated the preservation of life distribution classes under random maxima and minima and provided some counterexamples demonstrating that Li and Zuo (2004), Ahmad et al. (2005, 2006), and Kayid et al. (2011) have made a number of errors in some of their conclusions. These errors originate from the reliance on Equations (5) and (6), which are generally incorrect. We also showed that the positive life distribution classes $\operatorname{IFR}(2), \mathrm{NBU}(2), \mathrm{NBUS}, \mathrm{NBUT}$, NBUFR, NBAFR, and NBUCA are not preserved under neither random maxima nor random minima. Further, it was shown that the negative life distribution classes are also not preserved under random maxima. It should be noted that the conclusions made in this article are for different classes than those discussed by Hazra et al. (2014) and can, therefore, be considered an extension of Hazra's results to new aging classes.

\section{References}

Ahmad, I.A., Ahmed, A., Elbatal, I. and Kayid, M. (2006). An Aging Notion Derived from the Increasing Convex Ordering: the NBUCA Class. Jouranl of Statistical Planning and Inference. 136, 555-569.

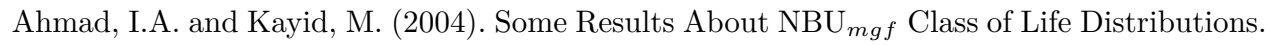
International Journal of Reliability and Applications. 5, 155-162.

Ahmad, I. and Kayid, M. (2007). Reversed Preservation of Stochastic Orders for Random Minima and Maxima with Applications. Statistical Papers. 48, 283-293.

Ahmad, I.A., Kayid, M. and Li, X. (2005). The NBUT Class of Life Distributions. IEEE Transection on Reliability, 54, 396-401.

Alzaid, A., Kim, J.S. and Proschan, F. (1991). Laplace Orderings and Its Applications. Journal of Applied Probability, 28, 116-130.

Barlow, R.E. and Proschan, F. (1975). Statistical Theory of Reliability and Life Testing. Holt, Rinehart, and Winston, New York. 
Bartoszewicz, J. (2001). Stochastic Comparisons of Rrandom Minima and Maxima from Life Distributions. Statistics \& Probability Letters, 55, 107-112.

Belzunce, F., Franco, M. , Ruiz, J. M. and Ruiz, M. C. (2001). On Partial Orderings between Coherent Systems with Different Structure. Probability in the Engineering and Informational Sciences, 15, 273-293.

Belzunce, F., Martinez, H.P. and Ruiz, J.M. (2007). Reversed Preservation Properties for Series and Parallel Systems. Journal of Applied Probability, 44, 928-937.

Boland,P. J., El-Neweihi,E. and Proschan, F. (1994). Applications of the Hazard Rate Ordering in Reliability and Order Statistics. Journal of Applied Probability, 31, 180-192.

Consul, P.C. (1984). On the Distributions of Order Statistics for a Random Sample Size. Statistica Neerlandica, 38, 249-256.

David, H.A. and Nagaraja, H.N.A. (2003). Order Statistics. New York: John Wiley \& Sons.

Denuit, M. (2001). Laplace Transform Ordering of Actuarial Quantities. Insurance: Mathematics and Economics, 29, 83-102.

Franco, M., Ruiz, J.M. and Ruiz, M.C. (2001). On Closure of the IFR(2) and NBU(2) Classes. Journal of Applied Probability, 38, 235-241.

Franco, M., Ruiz, M.C. and Ruiz, J.M. (2003). A Note on Closure of the ILR and DLR Classes under Formation of Coherent Systems. Sratistical Papers, 44, 279-288.

Hazra, N.K., Nanda, A.K. and Shaked, M. (2014). Some Aging Properties of Parallel and Series Systems with a Random Number of Components. Naval Research Logistics, 61, 238243.

Hendi, M.I., Mashhour, A.F. and Montasser, M. A. (1993). Closure of the NBUC Class under Formation of Parallel Systems. Journal of Applied Probability, 30, 975-978.

Hu, T. and He, F. (2000). A Note on Comparisons of k-out-of-n Systems with Respect to the Hazard and Reversed Hazard Rate Orders. Probability in the Engineering and Informational Sciences, 14, 27-32.

Jarrahiferiz, J., Mohtashami Borzadaran, G.R. and Rezaei Roknabadi, A.H. (2016). Properties of n-Laplace Transform Ratio Order and $L^{(n)}$-class of Life Distributions. REVSTAT Statistical Journal, 14, 229-244.

Kaas, R. and Hesselager, O. (1995). Ordering Claim Size Distributions and Mixed Poisson Probabilities. Insurance: Mathematics and Economics, 17, 193-201.

Kayid, M., Diab, L.S., Alzughaibi, A. and Ahmad, I.A. (2011). Starshaped Ordering of Life Distributions and Its Aging Properties. IEEE Transactions on Reliability, 60, 257-262. 
Klar, B. and Muller, A. (2003). Characterization of Classes of Lifetime Distributions Generalizing the NBUE Class. Journal of Applied Probability, 40, 20-32.

Kochar, S. (2012). Stochastic Comparisons of Order Statistics and Spacings: A Review. Int. Sch. Res. Netw, Probability and Statistics, Doi:10.5402/2012/839473.

Lai, C.D. and Xie, M. (2006). Stochastic Aging and Dependence for Reliability, Springer, New York.

Li, X. (2004). Some Properties of Ageing Notions Based on the Moment-Generating-Function Order. Journal of Applied Probability, 41, 927-934.

Li, X. and Lu, J. (2003). Stochastic Comparisons on Residual Life and Inactivity Time of Series and Parallel Systems. Probability in the Engineering and Informational Sciences, 17, 267-275.

Li, X. and Qiu, G. (2007). Some Preservation Results of NBUC Aging Property with Applications. Statistical Papers, 48, 581-594.

Li, X. and Yam, R.C.M. (2005). Reversed Preservation Properties of Some Negative Aging Conceptions and Stochastic Orders. Statistical Papers, 46, 65-78.

Li, X. and Zuo, M.J. (2004). Preservation of Stochastic Orders for Random Minima and Maxima, with Applications. Naval Research Logistics, 51, 332-344.

Miziula, P. (2012). Stochastic Orders and Ageing Classes. Mathematica Applicanda, 40, 105125.

Nanda, A.K., Misra, M., Paul, P. and Singh, H. (2005). Some Properties of Order Statistics when the Sample Size Is Random. Communications in Statistics - Theory and Methods, 34, 2105-2113.

Nanda, A.K. and Shaked, M. (2008). Partial Ordering and Aging Properties of Order Statistics when the Sample Size Is Random: a Brief Review. Communications in Statistics - Theory and Methods, 37, 1710-1720.

Pellerey, F. and Petakos, K. (2002). On Closure Property of the NBUC Class under Formation of Parallel Systems. IEEE Transaction on Reliability, 51, 452-454.

Salehi, E. and Tavangar, M. (2019). Stochastic Comparisons on Conditional Residual Lifetime and Inactivity Time of Coherent Systems with Exchangeable Components. Statistics and Probability Letters, 145, 327-337.

Shaked, M. (1975). On the Distribution of the Minimum and of the Maximum of a Random Number of iid Random Variables, in: G. P. Patil, S. Kotz, and J. K. Ord, (Editors), Statistical Distributions in Scientific Work, Vol. 1., Reidel, Boston, USA, 363-380.

Shaked, M. and Shanthikumar, J.G. (2007). Stochastic Orders. New York, Springer. 
Shaked, M. and Wong, T. (1997a). Stochastic Orders Based on Ratios of Laplace Transforms. Journal of Applied Probability, 34, 404-419.

Shaked, M. and Wong, T. (1997b). Stochastic Comparisons of Random Minima and Maxima. Journal of Applied Probability, 34, 420-425.

Zamani, Z., Mohtashami Borzadaran, G.R. and Amini, M. (2017). A Note on Some Preservation Results on the Laplace Transform Ordering of Residual Lives. Risk and Decision Analysis, 6, 225-229.

\section{Ebrahim Salehi}

Department of Industrial Engineering, Birjand University of Technology,

Birjand, Iran.

email:salehi@birjandut.ac.ir

\section{Ezzatollah Gholami}

Department of Basic Sciences,

Birjand University of Technology,

Birjand, Iran.

email: ezzatgholami@birjandut.ac.ir 